Proc. 19th Winter Workshop on

Nuclear Dynamics (2003) 000-000

19th Winter Workshop

on Nuclear Dynamics

Breckenridge, Colorado, USA

February 8-15, 2003

\title{
The Naive Parton Model and BRAHMS measurements
}

\author{
${ }^{1}$ Brookhaven National Laboratory, \\ Upton NY, USA
}

R. Debbe ${ }^{1}$ for the BRAHMS Collaboration

\begin{abstract}
The Naive Parton Model is revisited in conjunction with some data
\end{abstract} collected with BRAHMS spectrometers at RHIC

Keywords: BRAHMS, parton

PACS: 25.75.Dw

\section{Introduction}

While I studied the literature pertaining to work on the Color Glass Condensate, I realized that $I$ needed to have a good grasp of the parton model and found many references to Feynman's naive parton model. Among all of them, the lectures he gave at Stanford in 1971 Ref. [1] after returning from the Cornell International Symposium on Photon-Hadron Interactions, struck a chord in me because these lectures present his model in a very intuitive way, such that I can approach the subject even with my rudimentary theoretical training. As my reading went on I realized that I could use the data collected by the BRAHMS experiment at RHIC, as well as other published results, to present and discuss relevant sections of the lectures.

The lectures are organized in a general framework followed by three sets of hypothesis. I will follow a similar scheme, but I will only deal with the hypotheses that I consider to be closely related to the BRAHMS results.

\section{General Framework}

When Feynman describes the parton model he states that a model has a good chance of being correct if it starts by complying with the principles of quantum mechanics and relativity. Because of that, he goes on to say, field theory, besides its complicated nature, is the best tool to describe high energy systems.

In field theory, the wave function of a system can be written as an expansion or a linear combination of amplitudes to find "various kinds of bare field particles moving with various momenta". 
The wave function of a hadron with momentum $\mathrm{P}$ can be written as $|\Psi\rangle=$ $F^{*}|V A C\rangle$ with $|V A C\rangle$ being the wave function of the vacuum.

$$
F^{*}=\Psi_{0} \cdot 1+\Sigma_{p_{1}} \Psi_{1}\left(p_{1}\right) a_{p_{1}}^{*}+\frac{1}{2} \Sigma_{p_{1} p_{2}} a_{p_{1}}^{*} a_{p_{2}}^{*} \Psi_{2}\left(p_{1}, p_{2}\right)+\ldots . .
$$

The sum runs over the state of each system of partons characterized by the momentum of each parton $p_{i}$. For each term of the sum, whatever the number of partons, the condition $\Sigma p_{i}=P$.applies.

This is an expansion in Fock space. A similar technique is used to describe the state of a crystal where the creation operators $a^{*}$ represent individual phonon excitations, in this case $a_{p_{1}}^{*}$ creates a parton with momentum $p_{1}$ out of the vacuum.

This linear combination of systems of particles, with gradually increasing complexity, is a virtual system. It only goes "on shell" whenever a probe like a photon in Deep Inelastic Scattering couples to an individual parton and makes it real. The momentum of a particular type of parton will follow a distribution $f_{i}(x)$ (where i indicates the type of partons, much like the $u, d, s, c, b, t$, and gluons of today's QCD)

\section{Hypotheses of the naive parton model}

As said before, I will only focus on the hypotheses that allow some connection to BRAHMS results, as well as those that in my opinion are essential to the model.

\subsection{Hypothesis $A_{1}$}

The bulk of the products of a high energy interaction between two hadrons have transverse momentum smaller than $1 \mathrm{GeV} / \mathrm{c}\left\langle P_{T}^{2}\right\rangle \sim(0.4 \mathrm{GeV} / \mathrm{c})^{2}$. Most of the energy of the system after the collision is located in the forward fragmentation region. As $P \rightarrow \infty$ one can neglect any transverse component of the momentum of each parton.

\subsection{Hypothesis $A_{2}$}

As stated in section 2, the composite nature of the hadron description is meaningful only if there is a probe with sufficient energy. This hypothesis states that as $P \rightarrow \infty$ the probe can resolve the hadron to higher and higher detail, but beyond some high value of the probe's momentum, the distribution of partons reaches some sort of limiting state; the momentum of the partons is then distributed as fractions of the hadron momentum. The hadron is then described with distributions of partons that depend only on those fractions $\mathrm{x}$ where $p_{\text {parton }}=x P$. 




Fig. 1. a) A schematic of the parton density in rapidity for a hadron moving with high momentum $\mathrm{P}$ b) Schematic of the interaction of two hadrons $A$ and $B$. The top diagram shows the hadron A moving from left to right, the middle diagram shows hadron $B$ moving from right to left, and the bottom diagram shows the parton rapidity distribution of the final state.

\subsection{Hypothesis $A_{3}$}

Based on Deep Inelastic Scattering experimental results, where the structure function $F_{2}$ shows a constant behavior as $x \rightarrow 0$, it is conjectured that the probability to find a parton with momentum $\mathrm{x}$ between $\mathrm{x}$ and $x+d x$ follows a $\frac{d x}{x}$ trend near $\mathrm{x}=0$. (Here we use the fact that $F_{2} \sim x f(x)$ ).

More recent measurements at higher energies (e.g. H1 at HERA [7]) show that although the constant behavior is truly there at $Q^{2} \sim 0.4 \mathrm{GeV}^{2}$, at higher values of $Q^{2}, F_{2}$ rises as a power of $\mathrm{x}$ making the parton distribution behave rather like $\frac{1}{x^{1-\alpha}}$ with $0<\alpha<1$.

\subsection{Hypothesis $A_{4}$}

The density of partons in rapidity space $\left(y=y_{\text {hadron }}-\ln \left(\frac{1}{x}\right)\right)$ for a hadron moving at high momentum $P$ has the shape shown in figure 1a:

That distribution has three regions:

a) Near the hadron rapidity $\ln (2 \mathrm{P})$, where the momentum of the partons scale . with $P$, and where the number of partons is only dependent on $x$.

b) An intermediate region with a flat distribution that today would be related to the "sea quarks". 
b) The so called "wee" region where the momentum of the partons do not scale to the hadron momentum $\mathrm{P}$, and falls rapidly as $\frac{d p_{z}}{p_{z}}$. Where $p_{z}$ is the momentum of the parton along the total momentum of the hadron $P$.

The total number of partons in these distributions is mainly driven by the contribution under the flat region and is proportional to $\ln (2 \mathrm{P})$.

An analogy to a one dimensional drop of water $\ln (2 \mathrm{P})$ long is made to clarify this hypothesis. The most probable state is the one with a uniform distribution between both ends. Furthermore, the ends are independent of each other because they are separated by the long intermediate plateau.

\subsection{Hypothesis $A_{5}$}

The distribution of wees and plateau is the same for all hadrons.

As the two hadrons with $P \rightarrow \infty$ collide, each parton in a hadron is a probe of the distribution of partons of the second one. This hypothesis implies that what these partons "see" is a universal distribution. More recent theories establish a link between the scaling partons $(x \sim 1)$ and the small $\mathrm{x}$ partons; the high momentum partons radiate the ones at $x \sim 0$.

\subsection{Hypothesis $A_{6}$}

Figure $1 \mathrm{~b}$ can be used to state this hypothesis. Two hadrons A and B moving with high momentum $\mathrm{P}$ are shown in the two upper plots. After the collision, the rapidity distribution of the partons would be the following: At the most negative $y$ values the distributions are identical to those of the scaling region of hadron $B$ and only depend on $\mathrm{x}$.

At the highest positive rapidities, the distribution is identical to the scaling region of hadron $A$, and only depends on $\mathrm{x}$.

In the intermediate region the plateaus of the original hadrons fuses into one long plateau, and around $x \sim 0$ the $\frac{d P_{z}}{E}$ behavior of the "wee" regions compensate into a flat distribution that merges with the plateau mentioned above.

\subsection{Hypothesis $A_{7}$}

The partons from two colliding hadrons interact only if $\Delta y \leq 1$. This implies that heavy ion collisions are mainly interaction of the sea and wee parts of the initial hadrons and the $x \sim 1$ partons just continue forward.

The $x \sim 1$ regions on both hadrons will not interact and the shape of the distributions around $\ln (2 \mathrm{P})$ will only depend on the corresponding initial hadrons. This hypothesis is known today as "limiting fragmentation".

BRAHMS has measured charged particle production close to beam rapidities. Figure 2 shows the number of produced particles normalized to single nucleon system to make the comparison to proton anti-proton results from CERN.

Within the systematic error of the Au-Au ( 15\% at high $\eta)$, there is no 


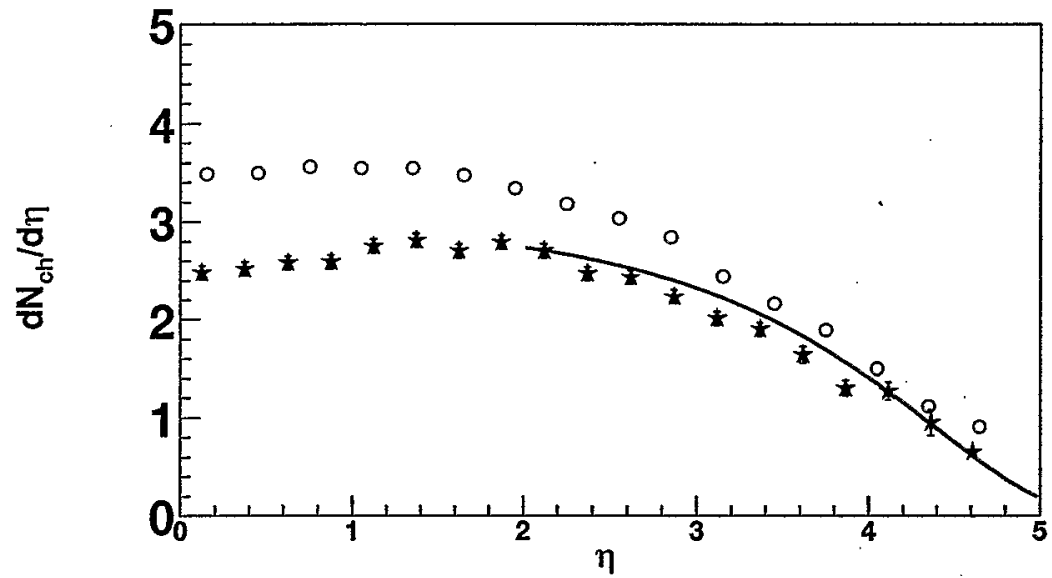

Fig. 2. BRAHMS charged particle and $p \bar{p}$ UA5 [5] as function of $\eta$. The curve is a parametrization of the form $a(1-x)^{b}$ where $b=3$. The errors on the Au-Au points are statistical only. Estimated systematic errors for that measurement are $8 \%$ at $y \sim 0$ and $10 \%$ at high $\eta$

difference between the BRAHMS and UA5 results at pseudo-rapidities greater than 4. One would conclude then, that limiting fragmentation is present for $\eta>4$, and as the curve included in the graph shows, the shape of the distributions are close to that of the parton distributions extracted from DIS data [8].

\subsection{Hypothesis $A_{8}$}

In average, the plateau region has the net value of any additive quantum numbers such as charge, third component of isospin, baryon number, etc equal to zero. If that was not the case, there will be a detectable increase of those quantities as a function of $\mathrm{P}$, maybe as $\ln (2 \mathrm{P})$ or $\ln (\mathrm{s})$ because it is assumed that the main contribution to the total multiplicity comes from the "plateau" of the rapidity density.

Compared to previous heavy ion systems studied at CERN and AGS, with RHIC energies the initial state of the collisions are closer to the "infinite momentum" frame that drives the main features of the parton model. We can thus check this hypothesis with BRAHMS data because of the wide rapidity coverage of this experiment.

Identified charged particle yields measured at different rapidities with the BRAHMS spectrometers [3] are used to test this hypothesis. To check the third component of isospin one would ideally use charged and neutral pions, but BRAHMS only measures charged pions. Figure 3a shows the $\pi^{+}-\pi^{-}$at all measured rapidities. The errors are statistical because the systematic errors are the same for both charges.

A similar test is done on total strangeness, this time kaon and $\Lambda$ are the main 
contributors. The net strangeness would then be $\left(K^{+}+\bar{\Lambda}\right)-\left(K^{-}+\Lambda\right)$. BRAHMS doesn't have a $\Lambda$ measurement but at $\mathrm{y}=0$ one can include the STAR most central value from Ref. [4]. Figure 3b shows that difference.
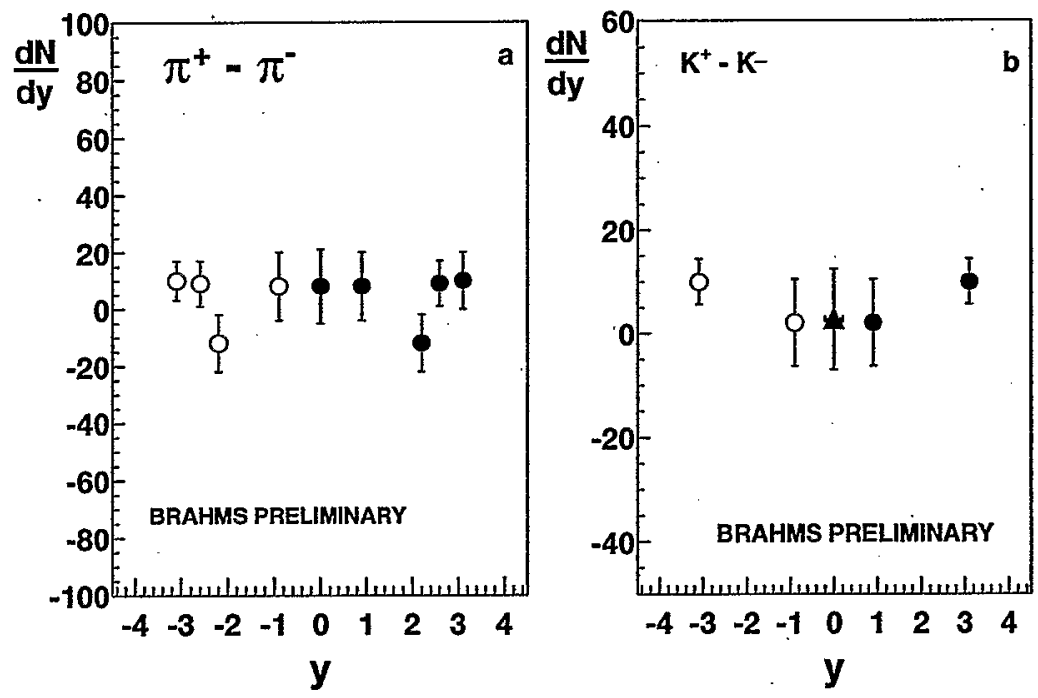

Fig. 3. Panel a shows the difference $\pi^{+}-\pi^{-}$as function of rapidity. Panel b shows the $K^{+}-K^{-}$difference. The point at $\mathrm{y}=0$ (filled triangle symbol) includes the $\Lambda$ measurement from STAR

Within errors, the pion difference is consistent with being equal to 0 all across the measured region $(|y|<3)$. The net strangeness within $(|y|<1)$ is also consistent with zero. At higher rapidities, where the $\Lambda$ contribution is not included, the difference grows indicating that the validity of hypothesis $A_{8}$ can not be tested with kaon data alone.

The net baryon should be tested on the yield of proton, neutron, and $\Lambda$ (as the yield of all other hyperons is small). BRAHMS doesn't have measurements of neutron or $\Lambda$ yields. The closest result would be the difference in yields of protons and anti-protons. Figure 4 shows the net proton as function of rapidity measured with the BRAHMS spectrometers [2].

The net baryon is clearly not what was expected by the Naive Parton Model.

\section{Summary}

Within the context of the Naive Parton Model an attempt was made at using some BRAHMS results to estimate how close was that model to a correct description of the systems that are now formed at RHIC in Au-Au collisions at $\sqrt{s_{N N}}=200 \mathrm{GeV}$. 


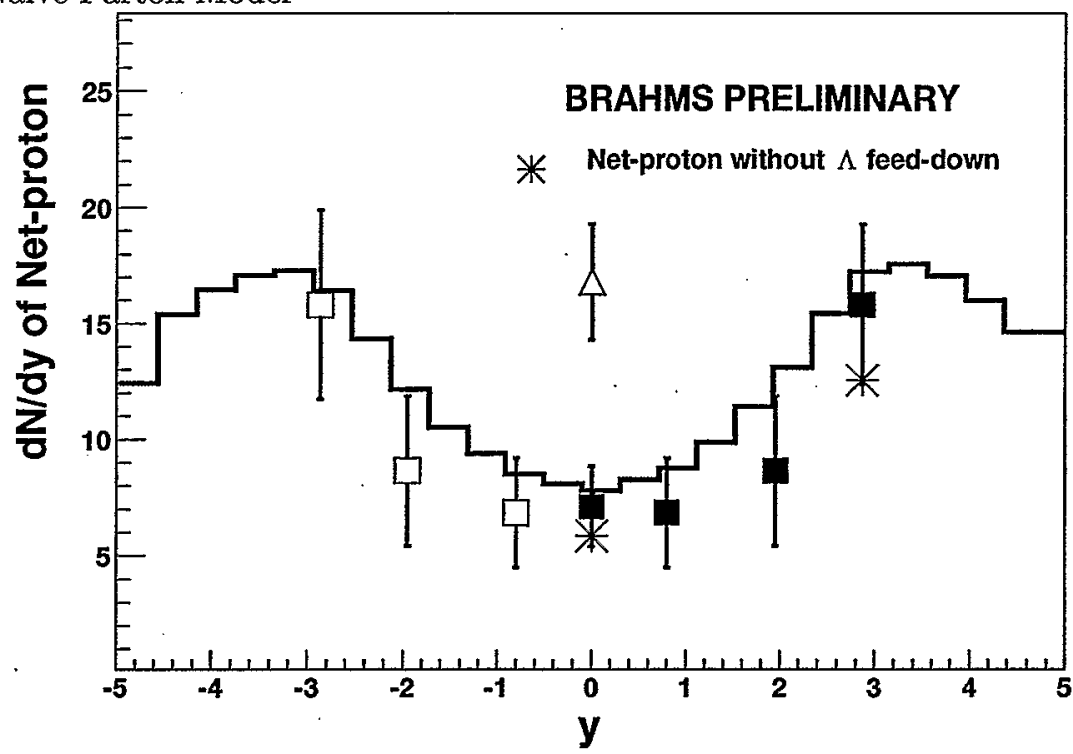

Fig. 4. Net proton as function of rapidity for Au-Au collisions at $\sqrt{s_{N N}}=$ $200 \mathrm{GeV}$. The contribution from $\Lambda$ decays was calculated at $\mathrm{y}=0$ and $\mathrm{y}=3$. The open triangle symbol includes the measured $\Lambda-\bar{\Lambda}$ measured by STAR [4] added to twice the net proton result to include the neutron contribution, in an attempt to show the net baryon content at mid-rapidity. The histogram, a simulation with HIJING/B [6], fits the net-proton data better than other models.

\section{Acknowledgment(s)}

This work was supported by the Division of Nuclear Physics of the Office of Science of the U.S. Department of Energy under contracts DE-AC02-98-CH10886, DEFG03-96-ER40981, and DE-FG02-99-ER41121 the Danish Natural Science Research Council, the Research Council of Norway, the Jagiellonian University Grants, the Romanian Ministry of Education and Research (5003/1999, 6077/2000).

\section{References}

1. R. P. Feynman Photon-Hadron Interactions Addison-Wesley Massachusetts ISBN 0-201-36074-8 (1998) 229.

2. J. H. Lee et al., Proceedings of Quark Matter 2002 Nantes France Nucl. Phys. A715 (2003) 482c-485c.

3. D. Ouerdane et al. Proceedings of Quark Matter 2002 Nantes France to be published in Nucl. Phys. A715 (2003) [nucl-ex/0212001].

4. C. Adler et al. Phys. Rev. Lett. 89092301 (2002)[nucl-ex/0203016].

5. G. J. Alner et al. Z. Phys. C 33 1-6 (1986).

6. S. E. Vance, M. Gyulassy X. N. Wang Nucl. Phys. A638 395c (1998). 
7. C. Adloff et al., Phys. Lett. B520 183 (2001) [hep-ex/0108035].

8. M. Glück et al., Eur. Phys. J. C5 461-470 (1998). 